\title{
CALCULATING GENERALISED IMAGE AND DISCRIMINANT MILNOR NUMBERS IN LOW DIMENSIONS
}

\author{
KEVIN HOUSTON \\ School of Mathematics, Leeds University, Leeds, LS2 9JT, U.K. \\ e-mail:khouston@amsta.leeds.ac.uk
}

(Received 2 July, 1999)

\begin{abstract}
Methods of calculating discriminant and image Milnor numbers in terms of the Milnor numbers of multiple point spaces are described for cases $f: \mathbb{C}^{3} \rightarrow \mathbb{C}^{4}$ and $F: \mathbb{C}^{3} \rightarrow \mathbb{C}^{3}$.

2000 Mathematics Subject Classification. 32S50, 32S55.
\end{abstract}

1. Introduction. In [4] Goryunov found the simple map-germs $f:\left(\mathbb{C}^{3}, 0\right) \rightarrow$ $\left(\mathbb{C}^{3}, 0\right)$. In [13] Marar and Tari calculated invariants associated to these maps. In particular they wished to find a relationship between the discriminant Milnor number, (this is the number of vanishing cycles in a local stabilisation of the map-germ) and the invariants they calculated. This paper grew out of finding the required relationship which is given in Remark 3.4.

In addition to discriminant Milnor number, one can investigate image Milnor numbers. Mond and others (see [3], [11], [16]), have generalised the notion of Milnor number to the case of the discriminant of a finitely $\mathcal{A}$-determined map-germ $f:\left(\mathbb{C}^{n}, 0\right) \rightarrow\left(\mathbb{C}^{p}, 0\right)$. For $n \geq p$ this is called the discriminant Milnor number; and for $n<p$, as the discriminant is the image of $f$, the number is called the image Milnor number.

One can also generalise by looking at the multiple point spaces in the target of the disentanglement map. It has been shown in [8] that these spaces are homotopically equivalent to a bouquet of spheres of dimension equal to the complex dimension of the space. Thus we can define what is called the $k$ th image Milnor number. To calculate these in the corank 1 case, we have Theorem 2.7 in the case $f:\left(\mathbb{C}^{2}, 0\right) \rightarrow\left(\mathbb{C}^{3}, 0\right)$ and Theorem 2.8 for $f:\left(\mathbb{C}^{3}, 0\right) \rightarrow\left(\mathbb{C}^{4}, 0\right)$.

One effect of defining these is that we get conditions on the source multiple point spaces. For example, in the case of finitely $\mathcal{A}$-determined $f:\left(\mathbb{C}^{3}, 0\right) \rightarrow$ $\left(\mathbb{C}^{4}, 0\right)$, if $D^{3}(f)$ is non-empty then $\mu\left(D^{3}(f)\right)=3 m+1$ for some $m$, see Remark 2.9 .

Formulae for calculating the invariants when the map is quasihomogeneous are given in Section 4.

2. Generalised Image Milnor Numbers. We will first make the necessary definitions and notations.

Let $f: X \rightarrow Y$ be a continuous map.

Definition 2.1. The $k$ th multiple point space, denoted by $D^{k}(f)$, is defined to be

$$
D^{k}(f):=\operatorname{closure}\left\{\left(x_{1}, \cdots, x_{k}\right) \in X^{k} \mid f\left(x_{1}\right)=\cdots=f\left(x_{k}\right), x_{i} \neq x_{j} \text {, for } i \neq j\right\} .
$$


There are continuous mappings $\varepsilon^{i, k}: D^{k}(f) \rightarrow D^{k-1}(f)$ defined by

$$
\varepsilon^{i, k}\left(\left(x_{1}, \cdots, x_{k}\right)\right)=\left(x_{1}, \cdots \hat{x}_{i}, \cdots, x_{k}\right)
$$

for $1 \leq i \leq k$.

The group of permutations on $k$ objects, denoted by $S_{k}$, acts on $D^{k}$ through its obvious permutation action on $X^{k}$.

Definition 2.2. Suppose that $M$ is a $\mathbb{Q}$-vector space upon which $S_{k}$ acts. Then the alternating part of $M$, denoted by $\mathrm{Alt}_{k} M$, is defined to be

$$
\mathrm{Alt}_{k} M:=\left\{m \in M \mid \sigma(m)=\operatorname{sign}(\sigma) m \text { for all } \sigma \in S_{k}\right\}
$$

Definition 2.3. Let $g: X \rightarrow Y$ be a map. The $k$ th image multiple point space of $g$, denoted by $M_{k}(g)$, is the closure in $Y$ of the set of points of $Y$ with $k$ or more preimages.

Assume that $f:\left(\mathbb{C}^{n}, 0\right) \rightarrow\left(\mathbb{C}^{n+1}, 0\right)$ is a finitely $\mathcal{A}$-determined corank 1 mapgerm, (we will abuse notation and also use $f$ to denote a representative of the germ $f$ ). Let $f_{t}: U_{t} \rightarrow \mathbb{C}^{n+1}$ be a stabilisation of $f$, where $U_{t}$ is an open domain in $\mathbb{C}^{n}$. That is, $f_{t}$ is $\mathcal{A}$-stable at every point.

Definition 2.4. The $k$ th disentanglement of $f$ is the closure of the set of points in the image of $f_{t} \cap B_{\varepsilon}$ (for some small ball $B_{\varepsilon}$ centred at zero) that have $k$ or more preimages. We denote this space by $\operatorname{Dis}_{k}(f)$.

One of the main theorems of $[8]$ says that if $f:\left(\mathbb{C}^{n}, 0\right) \rightarrow\left(\mathbb{C}^{n+1}, 0\right)$ is corank 1 and finitely $\mathcal{A}$-determined then $\operatorname{Dis}_{k}(f)$ is empty or is a bouquet of spheres of dimension $n-k+1$.

Definition 2.5. For $f:\left(\mathbb{C}^{n}, 0\right) \rightarrow\left(\mathbb{C}^{n+1}, 0\right)$ corank 1 and finitely $\mathcal{A}$-determined the $k$ th image Milnor number is the number of spheres in the bouquet of spheres of $\operatorname{Dis}_{k}(f)$. The number is denoted by $\mu_{I_{k}}$. We shall write $\mu_{I_{1}}(f)$ simply as $\mu_{I}(f)$.

This definition gives us a sequence of analytic invariants for the map $f$.

For a map the corank 1 hypothesis implies that the multiple point spaces of $f_{t}$ are Milnor fibres of the multiple point spaces of $f$ which are isolated complete intersection singularities (see [11]).

We denote by $\mu_{k}^{\text {alt }}(f)$ the dimension of the alternating cohomology group $\mathrm{Alt}_{k} H^{\operatorname{dim} D^{k}\left(f_{t}\right)}\left(D^{k}\left(f_{t}\right) ; \mathbb{Q}\right)$. Techniques for calculating this number from the Milnor numbers of the $D^{k}(f)$ and its restriction to reflecting hyperplanes are given in [9] (following [6] which contained an error). In particular we have

$$
\mu_{2}^{\text {alt }}(f)=\frac{1}{2}\left(\mu\left(D^{2}(f)\right)+\mu\left(D^{2}(f) \mid H\right)\right)
$$

where $H$ is the reflecting hyperplane given by permutation of coordinates in the ambient space, $X \times X$, for $D^{2}(f)$. 
Consider the spaces $D_{j}^{k}(\tilde{f})$ in $[6$, p. 55] for the map $\tilde{f}$. These are defined as the reduced image of $D^{k}(\tilde{f})$ in $D^{j}(\tilde{f})$ under any of the Cartesian projections. For example, $D_{j}^{k}(\tilde{f})$ is the image of

$$
\varepsilon^{1, j+1} \circ \varepsilon^{1, j+2} \circ \cdots \circ \varepsilon^{1, k-1} \circ \varepsilon^{1, k} .
$$

By Theorem 2.8 of the same paper these spaces have rational cohomology only in dimension equal to their complex dimension.

Denote by $\mu_{\Sigma_{k}}$ the dimension of $H^{n-k+1}\left(D_{1}^{k}\left(f_{t}\right)\right)$ for $2 \leq k \leq n+1$. Note that $\mu_{\Sigma_{k}} \neq \mu\left(D_{1}^{k}(f)\right)$ in general.

2.1. Calculating $\mu_{I_{k}}$ in terms of invariants of multiple point spaces. Suppose $f$ is of corank 1. Using the Milnor numbers of the multiple point spaces and the restrictions to the fixed point sets we can give formulae for $\mu_{I_{k}}$. We shall do this for the cases $n=2$ and $n=3$ but first a general result relating $\mu_{I_{2}}$ and $\mu_{I}$.

LEMMA 2.6 If $n \geq 2$, then $\mu_{I_{2}}=\mu_{\Sigma_{2}}-\mu_{I}$.

Proof. Consider the maps $f_{t}$ and $f_{t} \mid D_{1}^{2}\left(f_{t}\right)$. The multiple point spaces for these maps differ only in that their sources are different, the other multiple point spaces are the same by the explanation at the top of page 56 in [6]. Let $g: X \rightarrow Y$ be a finite continuous map and $g^{p}$ denote the map $g \mid D_{1}^{p}(g)$. Then, we have

$$
D^{j}\left(g^{p}\right)= \begin{cases}D_{j}^{p}(g) & \text { for } j<p \\ D^{j}(g) & \text { for } j \geq p .\end{cases}
$$

Thus, the first page of each of the image computing spectral sequences of [6, Proposition 2.3] differ only in the first column, the cohomology of the sources.

Down the $p+q=n+1$ anti-diagonal of both sequences we have the non-trivial alternating cohomology groups, (except $E_{1}^{0, n-1}$ ). The sum of the dimensions of these groups in the sequence is equal to $\mu_{I}$ since $E_{1}^{0, q}(f) \cong \mathbb{Q}$ for $q=0$ and trivial otherwise. The only other non-trivial terms for the sequence associated to $f_{t} \mid D_{1}^{2}\left(f_{t}\right)$ are $E_{1}^{0,0} \cong \mathbb{Q}$ and $E_{1}^{0, n-1}$ which has dimension $\mu_{\Sigma_{2}}$ by [6, Theorem 2.8]. Thus,

$$
1+(-1)^{n-1} \mu_{I_{2}}=\chi\left(\operatorname{Dis}_{2}\left(f_{t}\right)\right)=\chi\left(E_{1}^{*, *}\left(f_{t} \mid D_{1}^{2}\left(f_{t}\right)\right)\right)=1+(-1)^{n-1} \mu_{\Sigma_{2}}+(-1)^{n} \mu_{I} .
$$

2.1.1. The case $\boldsymbol{n}=2$. In the case $n=2$ there are three image Milnor numbers, $\mu_{I}=\mu_{I_{1}}, \mu_{I_{2}}$ and $\mu_{I_{3}}$. Since $\mu_{I}$ has been studied in [6] and [17], and when $D^{3}(f)$ is non-empty then $\mu_{I_{3}}$ is just $T-1$, the number of triple points in the image minus 1 , only the image Milnor number $\mu_{I_{2}}$ has not been studied thoroughly before.

Theorem 2.7. For $f:\left(\mathbb{C}^{2}, 0\right) \rightarrow\left(\mathbb{C}^{3}, 0\right)$ a corank 1 finitely $\mathcal{A}$-determined mapgerm, $\mu_{I_{2}}=\mu\left(D^{2}(f) / S_{2}\right)+2 T$, where $S_{2}$ is the group of permutations on two objects.

Proof. The space $D_{1}^{2}\left(f_{t}\right)$ is the image of the projection from $D^{2}\left(f_{t}\right)$ to the source of $f_{t}$ and the double point space of this mapping is homeomorphic to $D^{3}\left(f_{t}\right)$, a set of $6 T$ points. Thus, $\mu_{\Sigma_{2}}=\mu\left(D^{2}(f)\right)+3 T$.

Let us note that as the group $H^{\operatorname{dim}\left(D^{2}\right)}\left(D^{2}\left(f_{t}\right) ; \mathbb{Q}\right)$ decomposes into an alternating and symmetric part we get $\mu\left(D^{2}\right)=\mu^{\text {alt }}\left(D^{2}\right)+\mu\left(D^{2} / S_{2}\right)$. 
By Lemma 2.6 we have

$$
\begin{aligned}
\mu_{I_{2}} & =\mu_{\Sigma_{2}}-\mu_{I} \\
& =\left(\mu\left(D^{2}\right)+3 T\right)-\left(\mu_{2}^{\text {alt }}+\mu_{3}^{\text {alt }}\right) \\
& =\left(\mu_{2}^{\text {alt }}+\mu\left(D^{2} / S_{2}\right)+3 T\right)-\left(\mu_{2}^{\text {alt }}+T\right) \\
& =\mu\left(D^{2} / S_{2}\right)+2 T .
\end{aligned}
$$

2.1.2. Case $\boldsymbol{n}=3$. The topology of the map-germs from three-space to fourspace has been studied in [9] where $\mu_{I}$ has been calculated for all simple germs and germs of low codimension.

The group of permutations on $k$ objects acts on the ambient space, $X^{k}$, for $D^{k}(f)$ and this allows us to define reflecting hyperplanes in the ambient space. We shall denote a reflecting hyperplane in $D^{3}(f)$ by $H_{1}$, (it is irrelevant which hyperplane we choose). Again the precise details can be found in [6].

Theorem 2.8. For $f:\left(\mathbb{C}^{3}, 0\right) \rightarrow\left(\mathbb{C}^{4}, 0\right)$ a corank 1 finitely $\mathcal{A}$-determined mapgerm, the complete list of generalised image Milnor numbers is calculated in terms of invariants of multiple point spaces by the following.

(i) (cf. [6], [9] and [10]), $\mu_{I}=\frac{1}{2}\left(\mu\left(D^{2}\right)+\mu\left(D^{2} \mid H\right)\right)+\frac{1}{6}\left(\mu\left(D^{3}\right)+3 \mu\left(D^{3} \mid H_{1}\right)+2\right)$ $+Q$,

(ii) $\mu_{I_{2}}=\mu\left(D^{2} / S_{2}\right)+\frac{1}{3}\left(\mu\left(D^{3}\right)-1\right)+3 Q$,

(iii) $\mu_{I_{3}}=\frac{1}{6}\left(\mu\left(D^{3}\right)-3 \mu\left(D^{3} \mid H_{1}\right)+2\right)+3 Q$,

(iv) $\mu_{I_{4}}=Q-1$, (when $D^{4}(f)$ is non-empty),

where $Q$ is the number of quadruple points appearing in a stabilisation of $f$.

Proof. Part (i) is proved in the form above in [9] and in a different form in [10]. Part (iv) is trivial. For (ii) we will use Lemma 2.6. We have to calculate $\mu_{\Sigma_{2}}$, the second Betti number of $D_{1}^{2}\left(f_{t}\right)$. This space is the image of the projection $\varepsilon_{2_{t}}:=\varepsilon^{1,2}\left(f_{t}\right): D^{2}\left(f_{t}\right) \rightarrow U_{t}$, (define $\varepsilon_{2}$ to be $\left.\varepsilon_{2_{0}}\right)$. It is shown in [6, p. 55], that $D^{k}\left(\varepsilon_{2_{t}}\right)=D^{k+1}\left(f_{t}\right)$ for $k \geq 1$. Therefore using the image computing spectral sequence for $\varepsilon_{2_{t}}$ and [9, Theorem 2.6] we find,

$$
\begin{aligned}
\mu_{\Sigma_{2}} & =\mu\left(D^{1}\left(\varepsilon_{2}\right)\right)+\frac{1}{2}\left(\mu\left(D^{2}\left(\varepsilon_{2}\right)\right)+\mu\left(D^{2}\left(\varepsilon_{2}\right) \mid H\right)\right)+T\left(\varepsilon_{2}\right) \\
& =\mu\left(D^{2}(f)\right)+\frac{1}{2}\left(\mu\left(D^{3}(f)\right)+\mu\left(D^{3}(f) \mid H_{1}\right)\right)+4 Q .
\end{aligned}
$$

The calculation of $\mu_{I_{2}}$ is then straightforward:

$$
\begin{aligned}
\mu_{I_{2}} & =\mu_{\Sigma_{2}}-\mu_{I} \\
& =\frac{1}{2}\left(\mu\left(D^{2}\right)-\mu\left(D^{2} \mid H\right)\right)+\frac{1}{3}\left(\mu\left(D^{3}\right)-1\right)+3 Q \\
& =\mu\left(D^{2} / S_{2}\right)+\frac{1}{3}\left(\mu\left(D^{3}\right)-1\right)+3 Q .
\end{aligned}
$$

For part (iii) we use the image computing spectral sequence for the map $h_{1}=f_{t} \mid D_{1}^{3}$. Then $D^{2}\left(h_{1}\right)=D_{2}^{3}\left(f_{t}\right)$ and $D^{k}\left(h_{1}\right)=D^{k}\left(f_{t}\right)$ for $k \geq 3$, (top of page 56 of 
[6] again). From the image computing spectral sequence for $h_{1}$, [6, Theorem 2.8], we deduce that

$$
\mu_{I_{3}}=\mu_{\Sigma_{3}}-\operatorname{dim} \operatorname{Alt}_{2} H^{1}\left(D_{2}^{3}\left(f_{t}\right) ; \mathbb{Q}\right)+\mu_{3}^{\text {alt }}+\mu_{4}^{\text {alt }} .
$$

The value of $\mu_{3}^{\text {alt }}$ is shown to be $\frac{1}{6}\left(\mu\left(D^{3}\right)+3 \mu\left(D^{3} \mid H_{1}\right)+2\right)$ in [9], and $\mu_{4}^{\text {alt }}$ is equal to $Q$.

To calculate the alternating cohomology of $D_{2}^{3}\left(f_{t}\right)$ we need its first Betti number, $b_{1}\left(D_{2}^{3}\left(f_{t}\right)\right)$, and the number of points in the space $D_{2}^{3}\left(f_{t}\right) \mid H$ as the following shows. The alternating cohomology of $D_{2}^{3}\left(f_{t}\right)$ is calculated using [9, Theorem 2.7], where $\chi(Z)$ denotes the Euler Characteristic of the space $Z$ and $\chi^{\text {alt }}$ denotes the alternating version:

$$
\begin{aligned}
\chi^{\text {alt }}(Z) & =\frac{1}{2}(\chi(Z)-\chi(Z \mid H)) \\
-2 b_{1}^{\text {alt }}\left(D_{2}^{3}\left(f_{t}\right)\right) & =1-b_{1}\left(D_{2}^{3}\left(f_{t}\right)\right)-b_{0}\left(D_{2}^{3}\left(f_{t}\right) \mid H\right) \\
& =1-b_{1}\left(D_{2}^{3}\left(f_{t}\right)\right)-\left(\mu\left(D_{2}^{3}\left(f_{t}\right) \mid H\right)+1\right) \\
b_{1}^{\text {alt }}\left(D_{2}^{3}\left(f_{t}\right)\right) & =\frac{1}{2}\left(b_{1}\left(D_{2}^{3}\left(f_{t}\right)\right)+\mu\left(D_{2}^{3}\left(f_{t}\right) \mid H\right)\right) .
\end{aligned}
$$

The 0 th alternating homology group in the above is zero since $D_{2}^{3}\left(f_{t}\right)$ is connected and contains elements in the diagonal, hence any zero-cycle is homologous to a cycle in the diagonal and these are not alternating.

The set $D_{2}^{3}\left(f_{t}\right)$ is the image of the map $h_{2}: D^{3}\left(f_{t}\right) \rightarrow D^{2}\left(f_{t}\right)$. Thus $D^{1}\left(h_{2}\right)=D^{3}\left(f_{t}\right)$ and $D^{2}\left(h_{2}\right)=D^{4}\left(f_{t}\right)$. Hence, $b_{1}\left(D_{2}^{3}\left(f_{t}\right)\right)=\mu\left(D^{3}\right)+12 Q$. The set $D_{2}^{3}\left(f_{t}\right) \mid H$ has the same number of points as $D^{3}\left(f_{t}\right) \mid H_{1}$. Thus,

$$
\operatorname{dim} \operatorname{Alt}_{2} H^{1}\left(D_{2}^{3}\left(f_{t}\right) ; \mathbb{Q}\right)=\frac{1}{2}\left(\mu\left(D^{3}\right)+\mu\left(D^{3}\right) \mid H_{1}\right)+6 Q .
$$

To calculate $\mu_{\Sigma_{3}}$ we note that $D_{1}^{3}\left(f_{t}\right)$ is the image of the map $h_{3}: D_{2}^{3}\left(f_{t}\right) \rightarrow U_{t}$ and that $D^{k}\left(h_{3}\right)=D^{k+1}\left(f_{t}\right)$ for $k \geq 2$. From the spectral sequence for this map we deduce that

$$
\begin{aligned}
\mu_{\Sigma_{3}} & =b_{1}\left(D_{2}^{3}\left(f_{t}\right)\right)-\operatorname{dim} \mathrm{Alt}_{2} H^{1}\left(D^{3}\left(f_{t}\right) ; \mathbb{Q}\right)-\operatorname{dim} \mathrm{Alt}_{3} H^{0}\left(D^{4}\left(f_{t}\right) ; \mathbb{Q}\right) \\
& =\mu\left(D^{3}\right)+12 Q-\frac{1}{2}\left(\mu\left(D^{3}\right)+\mu\left(D^{3} \mid H_{1}\right)\right)-\frac{24}{6} Q \\
& =\frac{1}{2}\left(\mu\left(D^{3}\right)-\mu\left(D^{3} \mid H_{1}\right)\right)+8 Q .
\end{aligned}
$$

Part (iii) then follows from this as a straightforward calculation.

REMARK 2.9. Part (ii) proves, for a corank 1 finitely $\mathcal{A}$-determined $f:\left(\mathbb{C}^{3}, 0\right) \rightarrow\left(\mathbb{C}^{4}, 0\right)$, the intriguing observation made in [9], in the case of simple and low codimension germs, that $\mu\left(D^{3}(f)\right)=3 m+1$, for some $m$. Thus in particular $D^{3}(f)$ is either empty or singular for such maps.

REMARK 2.10. In both cases, $n=2$ and $n=3$, the alternating sum, $\sum_{k}(-1)^{k} \mu_{I_{k}}$ is a function of $\mu\left(D^{2}(f) \mid H\right)$. This may or may not be significant. 
Table 1 shows the generalised image Milnor numbers for the simple germs $f:\left(\mathbb{C}^{3}, 0\right) \rightarrow\left(\mathbb{C}^{4}, 0\right)$ and those in families of $\mathcal{A}_{e}$-codimension less than or equal to 4.

3. Map-germs $F:\left(\mathbb{C}^{3}, 0\right) \rightarrow\left(\mathbb{C}^{3}, 0\right)$. In [13] Marar and Tari classify the corank 1 simple map-germs from $\mathbb{R}^{3}$ to $\mathbb{R}^{3}$. By complexifying a real germ in the Marar and Tari list we can get a complex germ and hence can define the discriminant Milnor number as described in [3].

They pose the question of how the invariants arising from the multiple point spaces are related to the discrimimant Milnor number, i.e. the number of vanishing cycles in the stabilisation of the discriminant. The relation they require is described in our Remark 3.4. In order to relate the discriminant Milnor number to the invariants in [13, Table 1], we need to study two more invariants not in [13].

The first is the number of $A_{1} A_{2}$ points that appear in the discriminant under deformation of the original map. (An $A_{1} A_{2}$ point is the intersection of a plane and a cuspidal edge). For more general germs, i.e. non-simple ones, a further invariant is needed: the number of triple points that appear under deformation. As none of the simple germs produce triple points all the germs in [13, Table 1] have this number equal to zero.

3.1. Discriminant Milnor Numbers. Let $F:\left(\mathbb{C}^{3}, 0\right) \rightarrow\left(\mathbb{C}^{3}, 0\right)$ be a corank 1 finitely $\mathcal{A}$-determined map-germ. The critical point space of $F$, denoted $\Sigma$, is then a

Table 1: Generalised Image Milnor Numbers

\begin{tabular}{|c|c|c|c|c|c|}
\hline Singularity & Name & $\mu_{I}$ & $\mu_{I_{2}}$ & $\mu_{I_{3}}$ & $Q$ \\
\hline$\left(x, y, z^{2}, z\left(z^{2} \pm x^{2} \pm y^{k+1}\right)\right)$ & $A_{k}$ & $k$ & 0 & 0 & 0 \\
\hline$\left(x, y, z^{2}, z\left(z^{2}+x^{2} y \pm y^{k-1}\right)\right)$ & $D_{k}$ & $k$ & 0 & 0 & 0 \\
\hline$\left(x, y, z^{2}, z\left(z^{2}+x^{3} \pm y^{4}\right)\right)$ & $E_{6}$ & $k$ & 0 & 0 & 0 \\
\hline$\left(x, y, z^{2}, z\left(z^{2}+x^{3}+x y^{3}\right)\right)$ & $E_{7}$ & $k$ & 0 & 0 & 0 \\
\hline$\left(x, y, z^{2}, z\left(z^{2}+x^{3}+y^{5}\right)\right)$ & $E_{8}$ & $k$ & 0 & 0 & 0 \\
\hline$\left(x, y, z^{2}, z\left(x^{2} \pm y^{2} \pm z^{2 k}\right)\right)$ & $B_{k}$ & $k$ & $k-1$ & 0 & 0 \\
\hline$\left(x, y, z^{2}, z\left(x^{2}+y z^{2} \pm y^{k}\right)\right)$ & $C_{k}$ & $k$ & 1 & 0 & 0 \\
\hline$\left(x, y, z^{2}, z\left(x^{2}+y^{3} \pm z^{4}\right)\right)$ & $F_{4}$ & 4 & 2 & 0 & 0 \\
\hline$\left(x, y, y z+z^{4}, x z+z^{3}\right)$ & $P_{1,1}$ & 1 & 0 & 0 & 0 \\
\hline$\left(x, y, y z+z^{5}, x z+z^{3}\right)$ & $P_{1,2}$ & 2 & 1 & 0 & 0 \\
\hline$\left(x, y, y z+z^{6} \pm z^{3 k+2}, x z+z^{3}\right)$ & $P_{2,0}^{k}$ & $k+2$ & $2 k$ & $k-1$ & 0 \\
\hline$\left(z, y, y z+z^{7}+z^{8}, x z+z^{3}\right)$ & $P_{2,1}^{1}$ & 5 & 5 & 1 & 0 \\
\hline$\left(x, y, y z+z^{7}, x z+z^{3}\right)$ & $P_{2,1}$ & 5 & 5 & 1 & 0 \\
\hline$\left(x, y, x z+y z^{2}, z^{3} \pm y^{k} z\right)$ & $Q_{k}$ & $k$ & 0 & 0 & 0 \\
\hline$\left(x, y, x z+z^{3}, y z^{2}+z^{4}+z^{2 k-1}\right)$ & $R_{k}$ & $k+1$ & $k-1$ & 0 & 0 \\
\hline$\left(x, y, x z+y^{2} z^{2} \pm z^{3 j+2}, z^{3} \pm y^{k} z\right)$ & $S_{j, k}$ & $k+j+1$ & $2 j$ & $j-1$ & 0 \\
\hline$\left(x, y, y z+x z^{3} \pm z^{5}+a z^{7}, x z+z^{4}+b z^{6}\right)$ & I & 6 & 7 & 3 & 1 \\
\hline$\left(x, y, y z+x z^{3}+a z^{6}+z^{7}+b z^{8}+c z^{9}, x z+z^{4}\right)$ & II & 9 & 11 & 4 & 1 \\
\hline$\left(x, y, y z+z^{5}+z^{6}+a z^{7}, x z+z^{4}\right)$ & III & 6 & 7 & 3 & 1 \\
\hline$\left(x, y, y z+z^{5}+a z^{7}, x z+z^{4} \pm z^{6}\right)$ & IV & 6 & 7 & 3 & 1 \\
\hline$\left(x, y, x z+z^{5}+a y^{3} z^{2}+y^{4} z^{2}, z^{3} \pm y^{2} z\right)$ & $\mathrm{V}$ & 6 & 4 & 0 & 0 \\
\hline$\left(x, y, x z+z^{3}, y z^{2}+z^{5}+z^{6}+a z^{7}\right)$ & VI & 6 & 5 & 1 & 0 \\
\hline$\left(x, y, x z+z^{3}, y^{2} z+x z^{2}+a z^{4} \pm z^{5}\right)$ & VII & 6 & 3 & 0 & 0 \\
\hline$\left(x, y, x z+z^{4}+a z^{6}+b z^{7}, y z^{2}+z^{4}+z^{5}\right)$ & VIII & 8 & 8 & 3 & 1 \\
\hline
\end{tabular}


hypersurface. Since $F$ is stable outside the origin $\Sigma$ has an isolated singularity at the origin. (This includes the case where $\Sigma$ is non-singular!)

Let $f$ be the restriction of $F$ to $\Sigma$. Then $\Delta:=f(\Sigma)$ is the discriminant of $F$. We shall be interested in the change of the local topology of this space (and certain subspaces of it) under stabilisation of $F$. So suppose $F_{t}$ is a stabilisation of $F$. Then we can define $\Sigma_{t}$ as the critical point set of $F_{t}$ and define $f_{t}=F_{t} \mid \Sigma_{t}$ and $\Delta_{t}=f_{t}\left(\Sigma_{t}\right)$.

3.1.1. The spaces of interest. As in the case of maps from surfaces to three-space a good source of invariants is the set of invariants associated to the multiple point spaces of the map. We shall now describe these spaces and invariants carefully as there are a number of different meanings to the phrase 'double point space' in the literature.

The multiple point spaces we shall be interested are those defined by Goryunov in [5, Section 4]; they are also denoted by $D^{k}(f)$. In Mond's earlier definition of multiple point spaces, [15], a curve arising from the cuspidal edge in the image of $f$ would be included in $D^{2}(f)$. This is because the cuspidal edge points are of multiplicity 2, and so if one perturbs $f$ to make it $\mathcal{A}$-stable then a curve of double points appears. (Note that, in general, when $F$ is $\mathcal{A}$-stabilised $f_{t}$ is not even finitely $\mathcal{A}$ determined; this is due to the presence of the cuspidal edge in the image of $f$ ). Goryunov's definition of multiple point spaces allows one to remove this unwanted curve component.

We can also study the double point space in the source (this is the double point space that Marar and Tari study). Let $\mathcal{D}=D_{1}^{2}(f)$ and $\mathcal{D}_{t}=D_{1}^{2}\left(f_{t}\right)$ denote the relevant spaces. The invariant $\mu(d)$ of [13] is the Milnor number of $\mathcal{D}$.

One can also study the multiple point spaces in the image. Let $M_{k}\left(f_{t}\right)$ be the image of $D^{k}\left(f_{t}\right)$ in $\mathbb{C}^{3}$. We get $M_{2}\left(f_{t}\right)=f_{t}\left(\mathcal{D}_{t}\right)$, which is a connected complex curve by [2, Theorem 4.2.2].

Another space of interest is the preimage under $f_{t}$ of the cuspidal edge in the image. The invariant associated to this, $\mu(c)$, is studied in [13] but we shall be less interested in the cuspidal edge as it does not affect the topology of the discriminant, though note that it is useful in classification problems.

3.1.2. Invariants arising from stabilisation. As $F_{t}$ is stable the set $\Sigma_{t}$ is non-singular and so it is the Milnor fibre of $\Sigma$.

By [5, Theorem 5.3.2] $D^{2}\left(f_{t}\right)$ is the Milnor fibre of the isolated complete intersection curve singularity $D^{2}(f)$, and $D^{3}\left(f_{t}\right)$ is the Milnor fibre of the zero dimensional complete intersection singularity $D^{3}(f)$.

On each $D^{k}(f)$ there is an action of $S_{k}$ (the permutation group on $k$ objects). The fixed point set of $D^{2}(f)$ under the action of $S_{2}$, is a zero dimensional complete intersection. The fixed points of $D^{2}\left(f_{t}\right)$ under the action of $S_{2}$ correspond to swallowtails in the image. These are also known as $A_{3}$ points in the image. The orbits of $D^{3}\left(f_{t}\right)$ correspond to triple points in the image, i.e. the transverse crossing of three planes, denoted $A_{1}^{3}$. There is a third zero dimensional singularity, the transverse intersection of a cuspidal edge and a plane, an $A_{1} A_{2}$ point.

The double point set $\mathcal{D}_{t}$ is in general a singular curve. The singularities arise from the triple points and the $A_{1} A_{2}$ points in the image. A triple point in the image produces three nodes on $\mathcal{D}_{t}$ and an $A_{1} A_{2}$ produces a cusp.

3.1.3. The Invariants. The set $\Delta_{t}=M_{1}\left(f_{t}\right)$ is homotopically equivalent to a wedge of 2-spheres and the number of spheres is called the discriminant Milnor 
number, denoted $\mu_{\Delta}(F)$ (see [3]). As the set $M_{2}\left(f_{t}\right)$ is a connected complex curve we can define $\mu_{\Delta_{2}}(F)$ to be the number of circles in its homotopy type. We define $\mu_{\Delta_{3}}(F)$ to be the number of points in $M_{3}\left(f_{t}\right)$. We view these numbers as generalised discriminant Milnor numbers of $F$.

The invariants of the map and its stabilisation we wish to relate are

- $\mu_{\Delta}(F), \mu_{\Delta_{2}}(F)$, and $\mu_{\Delta_{3}}(F)$, the three generalised discriminant Milnor numbers,

- the Milnor numbers of $\Sigma, D^{2}(f), D^{2}(f) \mid H, D^{2}(f) / S_{2}, D^{3}(f)$ and $\mathcal{D}$,

- $\# A_{3}$, the number of swallowtails,

- \# $A_{1} A_{2}$, the number of intersections of planes and cuspidal edges, and

- $\# A_{1}^{3}$, the number of transverse crossings of three planes.

3.1.4. Relations between the invariants. The trivial relations between the invariants are $\# A_{3}=\mu\left(D^{2}(f) \mid H\right)+1$ and $\mu_{\Delta_{3}}=\# A_{1}^{3}=\frac{1}{6}\left(\mu\left(D^{3}(f)\right)+1\right)$.

Now, the main theorem for calculating the discriminant Milnor numbers is the following.

Theorem 3.1. For a corank 1 finitely $\mathcal{A}$-determined map-germ $F:\left(\mathbb{C}^{3}, 0\right) \rightarrow$ $\left(\mathbb{C}^{3}, 0\right)$ we have

(i) $\mu_{\Delta}(F)=\mu(\Sigma)+\frac{1}{2}\left(\mu\left(D^{2}(f)\right)+\mu\left(D^{2}(f) \mid H\right)\right)+\# A_{1}^{3}$;

(ii) $\mu_{\Delta_{2}}(F)=\mu\left(D^{2}(f) / S_{2}\right)+2 \# A_{1}^{3}$.

Proof. (i) The proof is the same as in the case of finitely $\mathcal{A}$-determined maps from surfaces to three-space but in this case we have a source with possibly nontrivial cohomology. See also [17].

(ii) We need to study the topology of $f_{t} \mid \mathcal{D}_{t}$. The multiple point spaces of this map are the same as $f_{t}$ for $k \geq 2$. In a similar way to Lemma 2.6 we find that

$$
\mu_{\Delta_{2}}=\operatorname{dim} H^{1}(\mathcal{D} ; \mathbb{C})-\frac{1}{2}\left(\mu\left(D^{2}\right)+\mu\left(D^{2} \mid H\right)\right)-\# A_{1}^{3} .
$$

Since the projection of $D^{2}\left(f_{t}\right)$ to $\mathcal{D}_{t}$ is injective except at the double points of the projection, of which there are $3 \# A_{1}^{3}$, we deduce that $\operatorname{dim}_{\mathbb{C}} H^{1}\left(\mathcal{D}_{t} ; \mathbb{C}\right)=$ $\mu\left(D^{2}(f)\right)+3 \# A_{1}^{3}$. From these two equations the equality of the theorem follows.

Table 2 shows the generalised discriminant Milnor numbers and the invariants not included in [13, Table 1].

REMARK 3.2. One entry in [13, Table 1] is incorrect. The Milnor number of $\mathcal{D}$ for $4_{2}^{k}$ is claimed to be $3 k+1$, however it is really $2 k-1$. The error occurs in that the

Table 2: Invariants for simple germs $F:\left(\mathbb{C}^{3}, 0\right) \rightarrow\left(\mathbb{C}^{3}, 0\right)$

\begin{tabular}{|c|c|c|c|c|c|c|}
\hline Name & Normal Form & $\mu_{\Delta}$ & $\mu_{\Delta_{2}}$ & $\mu_{\Delta_{3}}$ & $\mu\left(D^{2}\right)$ & $\# A_{1} A_{2}$ \\
\hline$A_{1}$ & $\left(x, y, z^{2}\right)$ & 0 & 0 & 0 & - & 0 \\
\hline$*$ & $\left(x, y, z^{3}+P(x, y) z\right)$ & $\mu(P)$ & 0 & 0 & - & 0 \\
\hline $4_{1}^{k}$ & $\left(x, y, z^{4}+x z \pm y^{k} z^{2}\right) k \geq 1$ & $k-1$ & 0 & 0 & $k-1$ & 0 \\
\hline $4_{2}^{k}$ & $\left(x, y, z^{4}+\left(y^{2} \pm x^{k}\right) z+x z^{2}\right) k \geq 2$ & $k+1$ & $k-1$ & 0 & $2 k-1$ & 0 \\
\hline 5 & $\left(x, y, z^{5}+x z+y z^{2}\right)$ & 1 & 0 & 0 & 1 & 2 \\
\hline $5_{2}$ & $\left(x, y, z^{5}+x z+y^{2} z^{2}+y z^{3}\right)$ & 3 & 1 & 0 & 4 & 3 \\
\hline $5_{3}$ & $\left(x, y, z^{5}+x z+y z^{3}\right)$ & 3 & 1 & 0 & 4 & 3 \\
\hline
\end{tabular}


authors have the set defined by $z=0$ and $y^{2}+x^{k}=0$ as a subset of $\mathcal{D}$. The equation $z=0$ makes the third component of the map equal to zero and this means that the points are not double points. If we remove this extraneous plane then we find that $\mathcal{D}$ is defined in $\mathbb{C}^{3}$ by $2 z^{2}+x=0$ and $y^{2}+x^{k}$ and so the value of $\mu(\mathcal{D})$ is $2 k-1$.

We now investigate the topology of the double point space in the source.

Lemma 3.3. For a map-germ as in the theorem above,

$$
\mu(\mathcal{D})=\mu\left(D^{2}\right)+2 \# A_{1} A_{2}+6 \# A_{1}^{3} .
$$

Proof. As $\mathcal{D}_{t}$ is a family of curves we can use [2, Theorem 4.2.2, part 2]. This gives that

$$
\mu(\mathcal{D})-\mu\left(\mathcal{D}_{t}\right)=\operatorname{dim}_{\mathbb{C}} H^{1}\left(\mathcal{D}_{t} ; \mathbb{C}\right),
$$

where $\mu\left(\mathcal{D}_{t}\right)$ is the sum of the Milnor numbers of the singularities of $\mathcal{D}_{t}$. But $\mu\left(\mathcal{D}_{t}\right)=3 \# A_{1}^{3}+2 \# A_{1} A_{2}$ as each $A_{1}^{3}$ point gives 3 nodes and each $A_{1} A_{2}$ point gives a cusp in $\mathcal{D}_{t}$.

The equality of the lemma follows from the description of $\operatorname{dim}_{\mathbb{C}} H^{1}\left(\mathcal{D}_{t} ; \mathbb{C}\right)$ given in the proof of the theorem.

REMARK 3.4. Using Lemma 3.3 and Theorem 3.1 we deduce that the relation required by Marar and Tari in [13] is

$$
\mu_{\Delta}(F)=\mu(\Sigma)+\frac{1}{2}\left(\mu(\mathcal{D})+\# A_{3}-1\right)-\# A_{1} A_{2}-2 \# A_{1}^{3} .
$$

REMARK 3.5. According to [5, Section 3.2] using the third coordinate function of $F$ and the defining equation of $\Sigma$ we can define the triple point space $D^{3}(F \mid \Sigma)$ in another way, using 5 equations in $\mathbb{C}^{5}$. Since only swallowtails, $A_{1} A_{2}$ points and triple points produce any points in this version of $D^{3}$ then the finite determinacy of $F$ means that $D^{3}(F \mid \Sigma)$, if it is non-empty, is an isolated complete intersection singularity of dimension zero.

Under stabilisation of $F, D^{3}(F \mid \Sigma)$ will split up so that swallowtails give fixed points, $A_{1} A_{2}$ points give orbits with 3 points and $A_{1}^{3}$ give free orbits, each orbit consisting of 6 points of course.

This means that

$$
\frac{\# D^{3}(F \mid \Sigma)}{6}=\# A_{3}+\# A_{2} A_{1}+\# A_{1}^{3} .
$$

This can provide a useful verification of calculations of the three invariants on the right hand side.

4. Quasihomogeneous maps. When the map $F$ is quasihomogeneous we can calculate the invariants using the map's weights and degrees. Formulae for calculating $\# A_{3}, \# A_{1} A_{2}$ and $\# A_{1}^{3}$ this way are given in [12]. The formula for $\mu(\Sigma)$ is easy 
to deduce as $\Sigma$ is an isolated hypersurface singularity (see [14]). Since $\mu\left(D^{2} \mid H\right)=\# A_{3}-1$ the only other formula needed to calculate $\mu_{\Delta}$ is for $\mu\left(D^{2}\right)$ and this is given in the next theorem. We also give a formula for calculating the degree of $D^{3}(F \mid \Sigma)$.

Theorem 4.1. Suppose $F$ is a finitely $\mathcal{A}$-determined map-germ of the form $F(x, y, z)=(x, y, g(x, y, z))$ and $g$ is quasihomogeneous of degree $d$ with weights $\left(w_{1}, w_{2}, w_{0}\right)$. Let $f$ be the restriction of $F$ to $\Sigma$, (this is the critical point space of $F$ ). Then

$$
\mu\left(D^{2}(f)\right)=1+\frac{\left(d-w_{0}\right)\left(d-2 w_{0}\right)\left(d-3 w_{0}\right)}{w_{0}^{2} w_{1} w_{2}}\left(3 d-\left(8 w_{0}+w_{1}+w_{2}\right)\right) .
$$

Proof. By [5], $D^{2}(f)$ is defined in $\mathbb{C}^{5}$ by equations of degree $d, d-w_{0}, d-2 w_{0}$ and $d-3 w_{0}$, where the weights are $w_{0}, w_{0}, w_{1}, w_{2}$ and $d$. The last arises from the equation $\lambda_{1}=g(x, y, z)$ in Goryunov's notation.

As $D^{2}(f)$ is a quasihomogeneous curve the Milnor number is easy to calculate from the weights and degrees (see [1, p. 36]).

TheOrem 4.2. Suppose $F$ is as in Theorem 4.1. Let $d_{1}$ denote the degree of $g$ and $d_{2}$ denote the degree of the equation defining $\Sigma$. Then

$$
\# A_{3}+\# A_{1}^{3}+\# A_{1} A_{2}=\frac{\left(d_{1}-w_{0}\right)\left(d_{1}-2 w_{0}\right) d_{2}\left(d_{2}-w_{0}\right)\left(d_{2}-2 w_{0}\right)}{w_{0}^{3} w_{1} w_{2}} .
$$

Proof. This follows from Remark 3.5 and an analysis of the weights and degrees of the equations defining $D^{3}(F \mid \Sigma)$ (see [5]).

\section{REFERENCES}

1. V. I. Arnol'd (editor), Dynamical Systems VIII, Encyclopaedia of Mathematical Sciences 39 (Springer-Verlag, Berlin, 1993).

2. R.-O. Buchweitz and G.-M. Greuel, The Milnor number and deformations of curve singularities, Invent. Math. 58 (1980), 241-281.

3. J. Damon and D. M. Q. Mond, $\mathcal{A}$-codimension and vanishing topology of discriminants, Invent. Math. 106 (1991), 217-242.

4. V. V. Goryunov, Singularities of projections of complete intersections, J. Sov. Math. 27 (1984), 2785-2811.

5. V. V. Goryunov, Semi-simplicial resolutions and homology of images and discriminants of mappings, Proc. London Math. Soc. 70 (1995), 363-385.

6. V. V. Goryunov and D. Mond, Vanishing cohomology of singularities of mappings, Compositio Math. 89 (1993), 45-80.

7. K. Houston, Image multiple point spaces and rectified homotopical depth, Proc. Amer. Math. Soc. 26 (1998) 323-331.

8. K. Houston, Bouquet and join theorems for disentanglements, Preprint, Middlesex University (1999).

9. K. Houston and N. P. Kirk, On the classification and geometry of corank 1 mapgerms from three-space to four-space, in Singularity Theory (London Math. Soc. Lecture Notes Series 263 (1999), ed. J. W. Bruce and D. M. Q. Mond), 325-351.

10. W. L. Marar, The Euler characteristic of the disentanglement of the image of a corank 1 map-germ, in Singularity Theory and its Applications (Lecture Notes in Mathematics 1462 (1991), ed. D. M. Q. Mond and J. Montaldi, Springer-Verlag, Berlin), 212-220. 
11. W. L. Marar and D. M. Q. Mond, Multiple point schemes for corank 1 maps, $J$. London Math. Soc. (2) 39 (1989), 553-567.

12. W. L. Marar, J. Montaldi and M. A. S. Ruas, Multiplicities of zero-schemes in quasihomogeneous corank 1 singularities, in Singularity Theory (London Math. Soc. Lecture Notes Series 263 (1999), ed. J. W. Bruce and D. M. Q. Mond), 353-367.

13. W. L. Marar and F. Tari, On the geometry of simple germs of corank 1 maps from $\mathbb{R}^{3}$ to $\mathbb{R}^{3}$, Math. Proc. Camb. Phil. Soc. 119 (1996), 469-481.

14. J. Milnor and P. Orlik, Isolated singularities defined by weighted homogeneous polynomials, Topology 9 (1970), 385-393.

15. D. Mond, On the classification of germs of maps from $\mathbb{R}^{2}$ to $\mathbb{R}^{3}$, Proc. London Math. Soc. (3) 50 (1985), 333-369.

16. D. Mond, Vanishing cycles for complex analytic maps, in Singularity Theory and its Applications (Lecture Notes in Mathematics 1462 (1991), ed. D. M. Q. Mond and J. Montaldi, Springer-Verlag, Berlin), 221-234.

17. D. Mond, Singularities of mappings from surfaces to 3 -space, in Proc. College Singularity Theory at Trieste 1991 (ed. D. T. Lê, K. Saito and B. Teissier, World Scientific, Singapore, 1995). 